\title{
Practical guidelines for postmortem examination and tissue sampling of cetaceans for ecotoxicological purposes
}

\author{
B. Clausen \\ Claus Nars Holm, 3520 Farum, Denmark
}

\section{INTRODUCTION}

Postmortem examinations of marine mammals are undertaken for a number of reasons, inter alia: to determine the possible cause of death; to obtain evidence of diseases or pathological changes; and to collect tissue and other samples (e.g. blood and organs) for further research. The latter can provide material for laboratory studies for a variety of subjects including toxicology, immunology, virology, parasitology and histopathology.

The collection of associated biological data such as sex, age, length and nutritional status from each specimen is an essential part of any postmortem examination. Without such data it is very difficult if not impossible to interpret the results of the aforementioned studies.

\section{PATHOLOGICAL EXAMINATIONS}

\section{Full scale autopsy guidelines}

Necropsies should preferably be carried out in an appropriate facility by a pathologist with experience of cetaceans. Several protocols for postmortem examinations have been developed. A good general field guide on dealing with stranded specimens is provided by Geraci and Lounsbury (1993). In addition, two more detailed sets of guidelines can be recommended. One is the extensive protocol for autopsies of marine mammals produced by Kuiken and Hartmann (1993). The other is a revised and updated version of this for cetaceans given in Law (1994). The latter is particularly relevant in the context of this volume, because as well as guidelines on postmortem examination, it also provides extensive information on the collection and storage of samples, analytical methods and quality control information for both age determination and the determination of trace metals and organochlorines.

Both protocols are comprehensive, detailed and require pathological expertise. However in many cases the conditions required to carry out such optimal, full scale necropsies (including sampling of material) cannot be met. It is for this reason that a more simple protocol is produced here.

\section{Necropsy field guide for laymen}

Common problems preventing comprehensive autopsies include the unavailability of a suitable pathologist; a lack of time; the lack of an adequate facility and equipment; the simultaneous stranding of several animals which must be examined in a short period; and the discovery of animals in remote areas from which transport is impossible. It is important to recognise that even in such cases, valuable information can be obtained by a more superficial examination and coarse sampling. 
Few tools are essential. These include for example: a knife, a whetstone and rubber gloves; tape measure; camera; notebook/tape recorder; containers for samples.

Serious life threatening infections have occurred among people cutting up marine mammals. Therefore gloves should be worn both for the handling of the animals as well as the necropsy. All wounds or should be carefully washed. Any sign of local infection in the days afterwards must be examined by a medical doctor.

\section{Stepwise postmortem examination and tissue sampling (Items marked with an asterisk are more important in the context of pollution studies)}

(1) *Before any cuts are made, the animal should be described, length and sex noted and the state of decomposition indicated (fresh, medium, rotten or falling apart). Although little further information may be obtained from animals in the latter two conditions, it is important because it provides clues for e.g. toxicologists on the suitability of samples for further analyses. Photographs can be extremely useful.

(2) *For odontocetes, at least four teeth should be collected for age determination. (Collection of earplugs for age determination of baleen whales is difficult and probably can only be undertaken by experienced personnel.)

(3) *Examine the animal for wounds, sores and abscesses, and signs of bycatch such as rope scars and flippers or flukes cut off. Even if unfamiliar with pathology the cutter may record large abnormailities like gunwounds, bycatch signs and abscesses etc. Again, photographs can be extremely useful.

(4) * Subsequently, where practical, place the animal on its side, cut through the blubber just over the middle, from the neck to the anus. The skin and the blubber can then be cut and dragged downwards. [Be careful with large whales - the pressure in the body cavity may be so high that the gases and outcoming rotten intestine may overturn the cutter!]. The organs should be taken out one by one, most easily in the following order.

(5) *The genital tract is cut loose from the behind and first loosened from the body when in females, the ovaries can be identified and collected (ovaries are particularly important as they can provide information on the reproductive history of the female). Testes are internal and are attached to the dorsal walls of the visceral cavity.

(6) The digestive tract, including the intestine, stomach, spleen and liver can also be removed from the anus area.. Organs are separated.

(7) Then the kidney and the adrenals in front of the kidneys can be identified up under the back and extracted.

(8) The diaphragm is cut, some ribs are removed and the lungs and heart are taken out and separated.

(9) Afterwards the main organs are examined and, if possible, weighed one by one.

(10) *Record whether the animal is pregnant or lactating, the latter is indicated by yellow-white fluid in the mammary tissue under the blubber on the belly. Special attention should be payed to not overlook small embryos.

(11) The kidneys are examined to see whether they appear similar and for any worms or infections.

(12) The intestine may be opened and the number of parasites estimated.

(13) The stomach is similarly investigated.

(14) The liver is cut and examined for nodules, abscesses and parasites.

(15) The heart is opened and examined for worms.

(16) So are the lungs, via the bronchi.

(17) The parasites are described in terms of e.g. tapeworms. roundworms and the total number estimated. Parasites can be preserved in alcohol (even in gin or whiskey). 
(18) *Tissue collected for toxicology should consist of at least a large $($ e.g. $10 \times 10 \mathrm{~cm})$ piece of blubber, muscle (if possible), liver and kidney.

(19) *A piece (ca $5 \times 5 \mathrm{~cm}$ ) of liver and of kidney should be separately put in a glass (e.g. a marmalade/jam jar rinsed beforehand with hot water).

(20) *A piece (ca $5 \times 5 \mathrm{~cm}$ ) of liver and kidney should be separately packed in plastic (bag or otherwise).

(21) *The labelled material should subsequently be preserved cold and as soon as suitable facilities are available, deep frozen.

The blubber (and sometimes muscle) is used to investigate the levels of the different types of lipophytic pollutants such as organochlorine compounds (PCBs, DDT and other pesticides). The liver and kidney are generally used to analyse levels of heavy metals such as mercury, cadmium and lead.

Without ignoring the toxic impact of heavy metals, it is generally agreed that of the known contaminants. organochlorines are the most likely compounds to affect cetaceans. They are globally widespread and may cause reproductive problems and lower the resistance against disease. Highest priority, therefore should be given to take at least blubber and muscle samples for organochlorine analysis.

Persons who have collected samples but are not in a position to have these analysed should approach the IWC or participants in the workshop, to obtain information on possible options for appropriate analyses to be carried out.

\section{ACKNOWLEDGEMENTS}

Thanks are due to the Workshop participants and in particular Alex Aguilar, Greg Donovan and Peter Reijnders.

\section{REFERENCES}

Geraci, J.R. and Lounsbury, V.J. 1993. Marine Mammals Ashore - A Field Guide For Strandings. Texas A\&M Sea Grant Publication, Galveston, Texas, USA. i-xi+305pp.

Kuiken. T. and Garcia Hartmann, M. 1993. Proceedings of the first European Cetacean Society workshop on cetacean pathology: Dissection techniques and tissue sampling. ECS Newsletter 17:1-39.

Law, R.J. 1994. Collaborative UK Marine Mammal Project: summary of data produced 1988-1992. Fisheries Research Technical Report 97, MAFF, Lowestoft, UK. 42pp. 